\title{
Discontinuation of Alprazolam After Successful Treatment of Panic Disorder: A Naturalistic Follow-up Study
}

\author{
James L. Abelson, M.D., Ph.D., AND George C. CuRTIS, M.D.
}

University of Michigan

\begin{abstract}
Low rates of benzodiazepine discontinuation have been seen in longterm outcome studies of alprazolam-treated panic disorder. Discontinuation studies reveal high rates of relapse when alprazolam is stopped. Available data may lack relevance to clinical practice, however, because drug taper rates are often more rapid than those used in many clinical settings. In order to obtain naturalistic data on the longterm course of alprazolam treatment of panic, we obtained follow-up data on 18 of 20 patients who had been enrolled one to two years earlier in a study of alprazolam effects on hypothalamic-pituitary-adrenal (HPA) axis functioning. The HPA protocol provided measures of symptom severity, biological markers, and short-term trearment response (12 weeks). Routine clinical care was then provided, with dosage adjustments dictated by individual clinical needs. The follow-up study repeated clinical measures at a mean of 21 months after initiation of treatment. Alprazolam was discontinued in $78 \%$ of patients. Relapse occurred in $36 \%$ of these. At follow-up, $61 \%$ were medication-free. Only $28 \%$ were on a benzodiazepine. The four patients remaining on alprazolam were on reduced doses and had maintained clinical gains. HPA axis activity did not predict dose or outcome. Factors related to successful alprazolam discontinuation are discussed.
\end{abstract}

Alprazolam is now an approved treatment for panic disorder, but discontinuation difficulties raise concerns about the long-term course of alprazolamtreated patients. Complications of alprazolam discontinuation include the following: (a) relapse, which is the recurrence of the anxiety symptoms for which treatment was originally sought; (b) rebound, which is relapse in which symptom intensity exceeds pretreatment baselines; and (c) withdrawal, which is the occurrence of specific symptoms associated with benzodiazepine discontinuation that were not present prior to treatment (Pecknold et al., 1988). Discontinuation studies have demonstrated high rates of all three phenomena

Patients described in this follow-up study entered treatment via a biological research protocol that was supported in part by a research grant from The Upjohn Company and in part by Clinical Research Center grant MO1RR00042. We are indebted to Pamela B. Schweitzer, M.S., for the excellent clinical care she provided to many of the patients.

Address correspondence to James L. Abelson, M.D., Ph.D., University of Michigan Medical Center/Box 0840, 1500 E. Medical Center Drive, Ann Arbor, MI 48109-0840. 
(e.g., relapse rates of $>74 \%$ ) during alprazolam taper (Fyer et al., 1987; Noyes et al., 1991). Data from discontinuation studies may lack relevance to clinical practice, however, because drug taper rates are dictated by protocol and are extremely rapid by clinical standards $(0.25-0.5 \mathrm{mg}$ reductions every three days). Slower rates and individualized taper programs may result in less relapse, rebound, and/or withdrawal.

Results of long-term outcome studies may be more relevant to clinical experience, but the limited data available from such studies also show low rates of successful discontinuation of the high-potency benzodiazepines used to treat panic disorder. In panic patients initially treated with alprazolam, follow-up at 1 to 2.5 years showed benzodiazepine discontinuation in less than 50\% (Nagy et al., 1989; Pecknold, 1990; Rickels, 1990). Follow-up of patients begun on alprazolam or clonazepam in a controlled treatment trial showed $73 \%$ still on a benzodiazepine and $78 \%$ still on medication 1.5 years later (Pollack et al., 1991). These data may suggest to some that obtaining drug-free remission of panic disorder is an unlikely outcome when treatment is initiated with benzodiazepines. In light of evidence from long-term follow-up of cognitive/behaviorally treated panic, which shows up to $81 \%$ of patients to be drugfree and panic-free at two years (Barlow, 1990), the benzodiazepine outcome data could be used to argue against the use of high-potency benzodiazepines in the treatment of panic. However, such conclusions may be unwarranted because the few available long-term outcome studies of benzodiazepine-treated panic were not designed to obtain drug-free remissions, and in some cases were specifically examining the results of long-term continuation of medication. Study goals and the availability of alternative treatment modalities are likely to significantly effect the rate of benzodiazepine discontinuation. If high-potency benzodiazepines have an appropriate place in our anti-panic armamentarium, then we need additional information on how successfully they can be discontinued. Higher rates of discontinuation should be possible when these medications are utilized in a setting in which the goal of treatment is to obtain drug-free, impairment-free, minimal symptom outcomes, and in which the intensity and modality of treatment can be flexibly altered in efforts to reach that goal. Documentation of reduced relapse rates and improved discontinuation rates would support the appropriate utilization of alprazolam in somc cases. Clinical guidelines that can facilitate discontinuation of alprazolam would be especially helpful.

We collected naturalistic data on the long-term course of alprazolam treatment of panic by conducting a follow-up study of patients who had been enrolled one to two years earlier in a study of alprazolam effects on hypothalamic-pituitary-adrenal (HPA) axis functioning. Entry into the follow-up study via the biological protocol insured that the patients were subjected to rigorous screening, standardized entry into treatment, and detailed clinical monitoring. It also facilitated examination of biological and clinical predictors of outcome. The taper and discontinuation phase was conducted naturalistically by clinicians skilled in the use of other medications and cognitive/behavioral techniques. Treatment goals included eventual discontinuation of medication and maintenance of a non-impaired, minimal symptom state. 


\section{METHOD}

Twenty patients with DSM-III-R defined panic disorder participated in the HPA study, which was begun in October, 1988. All were medically healthy, drug-free, and had no current or primary depression. They were diagnosed using a Structured Clinical Interview for DSM-III-R (SCID-UP) (Spitzer \& Williams, 1986). Assessment of HPA axis function included 24-hour corticotropin (ACTH) profiles (sampling every 15 minutes) and a corticotropinreleasing hormone (CRH) stimulation test (Roy-Byrne et al., 1986). Patients then entered a flexible-dose, open-label alprazolam protocol, beginning at a dose of $0.5 \mathrm{mg}$ tid and advancing slowly until panic attacks were controlled. Patients were monitored weekly, using the same instruments used in the Cross-National Collaborative Panic Study (Ballenger et al., 1988).

After 12 weeks of treatment, patients had repeat HPA axis assessment. They then transferred from the protocol to routine clinical care in which individualized treatment plans were developed. Alprazolam dose was adjusted as necessary to obtain or maintain full remission of panic symptoms. Adjunctive cognitive-behavioral therapy (CBT) was provided if significant panic or avoidance remained. New depressive episodes were treated with antidepressant medication. After one to three months of stable remission, alprazolam dose reductions were initiated. The treatment contract included the goal of eventual medication discontinuation, but discontinuation was not emphasized when dose reductions were initiated. These were presented as collaborative searches for the minimal dose needed to maintain remission. Patients retained control over the pace of reductions, but were cautioned to go no faster than $0.25 \mathrm{mg}$ every two weeks. Symptoms appearing during taper were used as opportunities for in vivo desensitization to somatic cues and as a context for practicing cognitive coping techniques. If relapse occurred, antidepressants or additional behavioral treatments were utilized.

In the fall of 1990 patients were re-interviewed. Treatment and illness course were obtained and clinical rating scales were repeated. Eighteen of the original twenty patients were interviewed. Two had lcft the state and could not be reached. Sixteen patients had received all follow-up care through our clinic. Nine were treated by the HPA study psychiatrist (JLA) with medication management and informal CBT as needed to manage reemergent symptoms. Two received similar treatment from a nurse clinician with four years of experience in providing multi-modal treatment to patients with anxiety disorders. Five were treated by the nurse clinician with medication management and formal CBT in either a structured group or individual setting. Two patients had transferred their care to psychiatrists closer to their homes. Variables used in data analyses are listed and described in Table 1. Descriptions of the clinical instruments can be found in Ballenger et al. (1988). Additional HPA axis variables were also analyzed, including 24hour secretory pulse frequency and amplitude and net ACTH response to $\mathrm{CRH}$, these same measures after 12 weeks of treatment, and the change in each HPA axis measure from before to during treatment. The results were identical regardless of measure used so, to conserve space, only the two variables listed in Table 1 are reported. 
TABLE 1

INSTRUMENTS AND MEASURES

\begin{tabular}{|c|c|}
\hline Variable & Description \\
\hline HAM-A & Total score on the Hamilton Anxiety Rating Scale. \\
\hline Agoraphobic avoidance & $\begin{array}{l}\text { Sum of avoidance scores on the } 6 \text { agoraphobia items of the } \\
\text { Marks-Sheehan Phobia Scale. }\end{array}$ \\
\hline Total disability & Sum of the subscale scores on the Sheehan Disability Scale. \\
\hline Panic attacks per week & $\begin{array}{l}\text { Average number of attacks per week over the prior month, } \\
\text { from the Panic Attack and Anticipatory Anxiety Scale. }\end{array}$ \\
\hline Composite Sxs & Sum of the scores on the above 4 clinical measures. \\
\hline Duration of taper & $\begin{array}{l}\text { Months until full discontinuation, counting from the first } \\
\text { reduction of alprazolam dose after completion of the HPA } \\
\text { protocol and achievement of stable remission. Duration was } \\
\text { adjusted for those patients still on alprazolam by adding the } \\
\text { additional time required to discontinue if tapered at the } \\
\text { maximal allowable rate }(0.25 \mathrm{mg} \text { every } 2 \text { weeks). }\end{array}$ \\
\hline Duration on drug & $\begin{array}{l}\text { Months from initiation of treatment with alprazolam to } \\
\text { discontinuation, adjusted as above for those still on drug. }\end{array}$ \\
\hline Relapse & $\begin{array}{l}\text { Recurrence of symptoms again meeting DSM-III-R criteria } \\
\text { for the diagnosis of panic disorder. }\end{array}$ \\
\hline ACTH-24h AUC & $\begin{array}{l}\text { Area under the } 24 \text { hour ACTH curve, prior to treatment, } \\
\text { calculated by trapezoidal approximation. }\end{array}$ \\
\hline Peak resp to $\mathrm{CRH}$ & $\begin{array}{l}\text { Peak ACTH level after CRH infusion minus the mean of the } \\
3 \text { samples prior to infusion (prior to initiation of treatment). }\end{array}$ \\
\hline
\end{tabular}

\section{RESIIITS}

Of the 18 patients completing the follow-up study, 10 were female and 8 male. Their mean $( \pm S D)$ age at entry was $30.2( \pm 5.8)$ years. They had been ill $6.1( \pm 8.3)$ years. They were having $3.5( \pm 2.7)$ panic attacks per week; and their mean Hamilton Anxiety Scale score was $15.3( \pm 7.8)$. Twelve had mild to moderate agoraphobia and six were not agoraphobic (by SCID). Nine had some history of other DSM-III-R Axis I diagnoses. Four had current or past generalized anxiety disorder. Two had histories of major depressive episodes and two of substance abuse (all in remission for $>6$ months). There was one patient with social phobia and one with a simple phobia (heights).

\section{Outcome}

After 12 weeks of treatment, patients were taking a mean alprazolam dose of $4.1 \mathrm{mg} /$ day. Substantial and significant clinical improvement had been obtained (see Table 2). The follow-up study repeated clinical measures 11 to 
TABLE 2

Clinical Status Before Treatment, After 12 WeEks on Alprazolam, and at Follow-up $(\operatorname{MEAN} \pm S D)$

\begin{tabular}{|c|c|c|c|c|}
\hline & \multirow[t]{2}{*}{ Pre-treatment } & \multirow[t]{2}{*}{ On drug } & \multicolumn{2}{|c|}{ Follow-up } \\
\hline & & & All subjects & Non-relapsers \\
\hline HAM-A & $15.3( \pm 7.8)$ & $6.4( \pm 5.7)^{* *}$ & $7.2( \pm 5.7) *$ & $5.3( \pm 4.3)$ \\
\hline $\begin{array}{l}\text { Agoraphobic } \\
\text { avoidance }\end{array}$ & $7.1( \pm 5.5)$ & $3.0( \pm 3.7) *$ & $2.1( \pm 3.5)^{* *}$ & $2.2( \pm 3.9)$ \\
\hline $\begin{array}{r}\text { Total } \\
\text { disability }\end{array}$ & $16.2( \pm 8.5)$ & $8.3( \pm 7.3)^{* *}$ & $5.4( \pm 4.0)^{* *}$ & $4.2( \pm 2.6) \dagger$ \\
\hline $\begin{array}{r}\text { Panic attacks } \\
\text { per week }\end{array}$ & $3.5( \pm 2.7)$ & $0.8( \pm 1.5)^{* *}$ & $0.6( \pm 0.9) * *$ & $0.2( \pm 0.6)$ \\
\hline
\end{tabular}

26 months after initiation of treatment (mean $=21$ months). Patients were treated with alprazolam for 4.5 to $>22$ months (mean adjusted duration $=11.6$ months). Drug tapers lasted from 1 to $>18$ months (mean adjusted duration $=$ 7.7 months). Alprazolam was discontinued in $78 \%$ of patients. Relapse occurred in $36 \%$ of these, an average of 6.4 months after drug discontinuation (range $=1$ to 18 months). At follow-up, $61 \%$ of patients were medication-free. Only $28 \%$ were on a benzodiazepine. No one who discontinued alprazolam restarted it. One patient was switched from alprazolam to clonazepam. All patients remaining on alprazolam $(n=4)$ were on substantially reduced doses. These four patients had reduced their alprazolam dose from a mean of 5.0 $\mathrm{mg} /$ day at 12 weeks to $2.1 \mathrm{mg} /$ day at follow-up. At follow-up they had been on drug for 18.8 months and had been tapering for 13.3 months. They had maintained their clinical gains (their mean composite symptom score at 12 weeks was 13.0 and at follow-up was 12.7). The total group remained significantly improved at follow-up, compared to pre-treatment, despite the presence of five relapsers; and non-relapsing patients showed significant additional reduction in disability between 12 weeks and follow-up (Table 2).

Relapse was defined as the recurrence of symptoms again meeting DSMIII-R criteria for panic disorder. All patients who relapsed were medicationfree at the time of relapse. Two relapsers were treated with an antidepressant (fluoxetine). One of these had a major depressive episode successully treated with nortriptyline during the follow-up period, but was med-free and not depressed at time of panic relapse; the other had significant dysphoric mood associated with her relapse. Both responded well to fluoxetine and CBT. The other three relapsers, one of whom also had a major depressive episode associated with her relapse, did not require resumption of medication and were successfully managed with CBT alone. One patient who did not have a relapse of her panic disorder also experienced a major depressive episode during the fol- 
low-up period, and was successfully treated with fluoxetine. None of the three patients $(17 \%)$ who developed major depressive episodes had a prior history of depression.

\section{Predictors of Outcome}

In order to explore outcome predictors we divided the patients into two groups: those who had achieved the goal of medication discontinuation without relapse ("Med-free/Remitted") and those who had not ("On-meds or Relapsed"), and compared these groups on clinical, biological, and treatment variables (see Table 3). The "Med-free/Remitted" group was doing quite well at follow-up - they were nearly panic-free (one of them had a single panic attack in the month prior to the follow-up interview) and had an extremely low mean HAM-A score (see Table 3). The "On-meds or Relapsed" group was significantly more symptomatic, but even their mean HAM-A score and panic attack frequency (Table 3) were low relative to those reported in other outcome studies (Nagy et al., 1989; Barlow, 1990; Pecknold et al., 1988). Though they differed in clinical status at follow-up, the groups did not differ signifi-

TABLE 3

Comparison of the Two Outcome Groups on Clinical, Drug, and HPA Variables

\begin{tabular}{lcccc}
\hline & $\begin{array}{c}\text { Med-free/Remitted" } \\
(\mathrm{n}=7)\end{array}$ & $\begin{array}{c}\text { "On-meds or Relapsed" } \\
(\mathrm{n}=11)\end{array}$ & $\mathrm{t} *$ & $\mathrm{p}$ \\
\hline Composite Sxs (Pretreatment) & $35.0( \pm 19.7)$ & $46.5( \pm 21.8)$ & 1.14 & .27 \\
Composite Sxs (12 wks) & $18.4( \pm 16.1)$ & $18.7( \pm 13.3)$ & .04 & .97 \\
Composite Sxs (follow-up) & $8.8( \pm 7.9)$ & $18.3( \pm 10.6)$ & 2.03 & .06 \\
HAM-A (follow-up) & $3.7 \pm( \pm 2.9)$ & $9.6( \pm 6.1)$ & 2.36 & .03 \\
Disability (follow-up) & $2.7( \pm 1.5)$ & $7.2( \pm 4.1)$ & 2.72 & .02 \\
Panic attacks/wk (follow-up) & $0.1( \pm 0.2)$ & $0.9( \pm 1.1)$ & 1.87 & .08 \\
ACTH-24h AUC (py/min ${ }^{*}$ Inin) & $23464( \pm 6003)$ & $21858( \pm 10385)$ & .37 & .72 \\
Peak resp to CRH (pg/ml) & $36.9( \pm 16.6)$ & $45.9( \pm 30.1)$ & .72 & .48 \\
Alprazolam dose (12 wks)(mg) & $2.6( \pm 1.3)$ & $5.0( \pm 1.8)$ & 3.10 & .007 \\
Duration of taper (mos.) & $3.2( \pm 1.9)$ & $10.4( \pm 6.3)$ & 2.90 & .01 \\
Duration on drug (mos.) & $6.3( \pm 1.8)$ & $15.0( \pm 7.2)$ & 3.11 & .007 \\
Age at time of study (yrs.) & $28( \pm 7.4)$ & $31.6( \pm 4.2)$ & 1.34 & .20 \\
Age at panic onset (yrs.) & $18.3( \pm 6.6)$ & $28( \pm 4.0)$ & 3.9 & .001 \\
\hline
\end{tabular}

* un-paired l-tests with of $=16$ for all variables except HAM-A and Peak resp to $\mathrm{CRH}$, where $\mathrm{df}=15$ 
cantly in clinical status prior to treatment. The "On-meds or Relapsed" group had higher scores on the composite symptom index (Table 3) and each of the clinical scales making up this index, but none of these group differences reached significance. After 12 weeks of treatment, the two groups had nearly identical scores on all of the clinical variables. The groups were nearly identical on all measures of HPA activity, both before treatment and after 12 weeks on medication. The "On-meds or Relapsed" group was on a higher dose of alprazolam at 12 weeks and tapered more slowly, remaining on drug significantly longer than the "Med-free/Remitted" group. The "On-meds or Relapsed" group also had a greater age of onset of their panic disorder, but did not differ in age at time of study.

Correlational analyses confirmed the relationship between alprazolam dose and outcomc, with significant positive correlations between dose at 12 weeks and follow-up HAM-A score $(r=.74, p=.0007)$, panic attack frequency $(r=.69, p=.002)$, and disability $(r=.71, p=.0009)$. Dose was not related to clinical severity prior to treatment and was not related to HPA axis variables $(r<.37, p>.12$ for all variables).

Chi-square analyses revealed no relationship between outcome and the presence or absence (current or past) of other Axis I disorders $\left(\chi^{2}(1)=.23\right.$, $p=.63)$. "Med-free/Remitted" status was related at a trend level to the absence of SCID-diagnosed agoraphobia $\left(\chi^{2}(1)=2.92, p=.09\right.$ ). Sixty-seven percent of six non-agoraphobics were medication-free and non-relapsed at follow-up, compared to $25 \%$ of the 12 agoraphobics.

Difficulty in discontinuing alprazolam (duration of taper) was not related to any measure of clinical, drug, or HPA status. Age of onset of panic disorder was related to duration of taper $(r=.55, p=.02)$.

\section{DISCUSSION}

Twenty-one months after initiation of alprazolam treatment, $72 \%$ of our patients had discontinued use of benzodiazepines. In other published studies, only $27 \%-47 \%$ of patients were off high-potency benzodiazepines at 12 to 30 month follow-up (see Table 4). Despite our high rate of benzodiazepine discontinuation, our relapse rate was similar to that seen following cessation of non-benzodiazepine drug treatment of panic disorder (Mavissakalian, 1982; Zitrin et al., 1980); and levels of residual symptomatology in non-relapsing medication discontinuers were quite low. Though it is difficult to compare levels of residual symptomatology across studies, our "Med-free/Remitted" group was doing well at follow-up by any available standards of panic disorder outcome research (0.1 panic attacks/week, six of the seven patients panicfree, and a mean HAM-A of 3.7). Even the total patient group (including the five relapsers) had a fairly low mean HAM-A score and panic attack rate at follow-up. The following factors may have contributed to successful benzodiazepine discontinuation: (a) the use of cognitive-behavioral techniques to treat residual and recurrent symptoms; (b) the use of antidepressant medications when a return to drug treatment was necessary; (c) an explicit goal, agreed upon between patient and clinician, to work towards achievement of drug- 
TABLE 4

Long-Term Outcome Studies of High-Potency Benzodiazepine Treatment of Patients with PANIC Disorder

\begin{tabular}{lccccccc}
\hline & & & & & \multicolumn{3}{c}{ Follow-up } \\
\cline { 7 - 8 } Author & $\mathrm{n}$ & benzodiazepine & $\begin{array}{c}\text { stopped } \\
\text { in: }\end{array}$ & $\begin{array}{c}\text { relapse } \\
\text { rate }\end{array}$ & $\begin{array}{c}\text { duration } \\
\text { (mos.) }\end{array}$ & On drug & $\begin{array}{c}\text { On } \\
\text { benzodiazepine }\end{array}$ \\
\hline Nagy et al '89 & 60 & alprazolam & $?$ & $?$ & 30 & $70 \%$ & $70 \%$ \\
Pecknold '90 & 142 & alprazolam & $66.9 \%$ & $29 \%$ & $?$ & $52.8 \%$ & $52.8 \%$ \\
Rickels '90 & 27 & alprazolam & $70 \%$ & $37 \%$ & 14 & $56 \%$ & $56 \%$ \\
Pollack et al '86 & 50 & clonazepam & $42 \%$ & $?$ & 12 & $58 \%$ & $54 \%$ \\
Pollack et al '91 & 59 & both & $?$ & $?$ & 18 & $78 \%$ & $73 \%$ \\
\hline
\end{tabular}

free, impairment-free, minimal-symptom outcomes; and (d) an approach to that goal that emphasized slow taper rates, patient control, and minimization of symptoms rather than focussing on discontinuation.

Our sense of success is tempered by the fact that at follow-up, 11 of 18 paticnts had not achicved the goal of medication-free remission. Nearly two years after coming to the clinic, the majority of patients remained in need of ongoing treatment. This may reflect the chronic nature of panic disorder, or remaining inadequacies in our treatments. It is noteworthy, in light of recent recommendations for long-term medication continuation in panic disorder (Ballenger, 1992), that no relapses occurred while patients remained on medication. However, it is also noteworthy that the majority of the relapses were successfully managed without a return to medication and none were refractory to treatment. Our patients appeared to do well compared to those in other follow-up studies of benzodiazepine-treated panic disorder (Nagy et al., 1989; Pecknold, 1990; Rickels, 1990; Pollack et al., 1991); but they did not do as well as patients in follow-up studies of CBT-treated panic (Barlow, 1990). Utilization of CBT may have improved our drug discontinuation rate and CBT alone may produce even better results; but it is risky to compare outcomes across studies done in different centers, utilizing varied methodologies and perhaps differing patient populations. Differences in initial symptom severity are particularly relevant, are difficult to control across studies, and may confound attempts to compare our outcomes to those of others. Though initial symptom severity did not predict outcome within our study, a relationship might have emerged with a larger sample. Our impression from this naturalistic study that our approach to alprazolam discontinuation facilitated improved long-term outcomes can only be definitively tested in a large-scale controlled trial.

Although our study is not directly comparable to benzodiazepine discontinuation studies (which use structured taper protocols to examine the immediate effects of discontinuation), we believe that our patients experienced considerably less subjective distress during taper than those patients described in the 
discontinuation literature. Many patients tapering alprazolam in structured protocols report that during the taper their anxiety symptoms became worse or much worse than they had been prior to treatment (Noyes et al., 1991). Published protocols, however, use rapid dose reduction schedules $(\geq 0.5 \mathrm{mg}$ every three days), and authors have hypothesized that slower schedules would result in less distress (Noyes et al., 1991). Our patients did report significant distress associated with dose reductions, especially in the first one to five days after each reduction, but they were taught to cognitively block the development of this distress into full-blown panic attacks by properly attributing them to the dose reduction. They were encouraged to use their distress as an opportunity to begin to desensitize themselves to anxiety-associated somatic cues. Many patients also reported that their ability to control the timing of dose reductions, so they could most comfortably fit the period of increased distress into their daily schedules, made it easier to move forward with further reductions. The fact that the four patients who were in the midst of drug tapers at the time of follow-up were experiencing no increase in symptoms above their pre-taper, remitted states supports the hypothesis that slower, individualized taper programs do not lead to the substantial increase in symptoms and subjective distress seen with structured taper protocols.

We were able to identify only two factors that predicted better outcome: (a) a lower dose of alprazolam after 12 weeks of treatment; and (b) a lower age of onset of the disorder. The absence of agoraphobia may also predict better outcome. The link between dose and outcome could be partly due to the way we dichotomized outcome and included patients still on alprazolam at follow-up in the "On-meds or Relapsed" group. Higher doses at entry into follow-up might be expected to be linked to longer duration of tapers and increased likelihood of remaining on drug at follow-up. However, the direct correlation between dose at 12 weeks and duration of taper was not significant, whereas dose at 12 weeks was highly correlated with clinical status at 21 months. This suggests that those patients who required higher doses of alprazolam to initially control their symptoms did have poorer clinical outcomes in the long run. Surprisingly, this was not a function of initial symptom severity, which was not related to clinical outcome or alprazolam dose requirements. Factors such as panic subtypes (agoraphobic/nonagoraphobic), and Axis I and Axis II comorbidity have been linked to clinical status in long-term follow-up of panic patients (Noyes et al., 1990), and may mediate the link between dose required for initial control of symptoms and long-term outcome. Our data did support a link between agoraphobia and poorer outcome, but not between Axis I comorbidity and outcome. However, our sample size may have been too small to detect this latter association. We have no data on Axis II diagnoses in our patients. Additional research will be needed to identify more clearly the factors that determine initial dose requirements and link this to long-term outcome. Clinically, the data suggest a need to attend to factors such as phobic avoidance and comorbidity, and to consider other treatment modalities before pushing alprazolam to upper-range dosage levels.

Reasons for a link between an older age of onset of panic disorder and greater difficulty in discontinuing alprazolam are not immediately apparent. Though group differences in duration of illness were not significant, the 
"poorer" outcome patients surprisingly had more recent onset of their disorder. This finding may be an anomaly of our small sample, and further research is needed to verify and explore this potential predictive factor.

There is some prior evidence that increased HPA activity is associated with increased risk of relapse upon alprazolam discontinuation (Coryell et al., 1989) and increased symptomatology and disability at long-term follow-up (Coryell et al., 1991). We were not able to verify a link between HPA axis activity and clinical status at follow-up, despite very detailed study of the HPA axis.

We conclude that in the context of a comprehensive anxiety management program, alprazolam can be used effectively, without abuse or tolerance in the sense of a need for escalating doses, and with successful discontinuation without a rate of relapse in excess of that seen with other, drugs. Given the inherent limitations of a naturalistic study, more extensive conclusions cannot be drawn. However, we believe that alprazolam need not be excluded from our anti-panic armamentarium. Its most appropriate role remains to be defined, given the availability of other pharmacological and non-pharmacological treatments; but we believe that it can be most useful when rapid, efficient control of acute symptoms is needed. We believe that if alprazolam is not effective in its lower dose range (1-3 mg/day), clinicians should consider adding an antidepressant medication and/or utilizing cognitive-behavioral techniques before pushing the alprazolam to higher dose levels. A useful approach to tapering alprazolam after successful treatment is to initiate a collaborative search for the minimal effective dose, allowing patients control over the taper rate, reducing no faster than $0.25 \mathrm{mg} / 2$ weeks, treating symptoms that occur during taper as opportunities for desensitization to somatic cues, and utilizing antidepressants and/or behavioral treatment for symptom recurrence. Long-term outcomes may be enhanced by the ability to apply multiple modalities flexibly, including cognitive/behavioral tcchniques. Explicit attention to "comorbid factors," including environmental and intrapsychic issues as well as panic subtypes and other Axis I and Axis II diagnoses, may also facilitate improved long-term outcomes. Research is needed to help us better match our growing arsenal of treatment techniques to the specific needs of individual patients.

\section{REFERENCES}

Ballenger, J. C., Burrows, G. D., DuPont, R. L., Lesser, I. M., Noyes, R., Pecknold, J. C., Rifkin, A., \& Swinson, R. P. (1988). Alprazolam in panic disorder and agoraphobia: Results from a multicenter trial. Archives of General Psychiatry, 45, 413-422.

Ballenger, J. C. (1992). Update on panic disorder: Termination of therapy issues. Presented at the Anxicty Disorders Association of America 12th National Conference, Houston, Texas, April 11, 1992.

Barlow, D. H. (1990). Long-term outcome for patients with panic disorder treated with cognitivebehavioral therapy. Journal of Clinical Psychiatry, 51[12, Supp. A], 17-23.

Coryell, W., Noyes, R., \& Reich, J. (1991). The prognostic significance of HPA-axis disturbance in panic disorder: A three-year follow-up. Biological Psychiatry, 29, 96-102.

Coryell, W., Noyes, R., \& Schlechte, J. (1989). 'The significance of HPA axis disturbance in panic disorder. Biological Psychiatry, 25, 989-1002. 
Fyer, A. J., Liebowitz, M. R., Gorman, J. M., Campeas, R., Levin, A., Davies, S. O., Goetz, D., \& Klein, D. F. (1987). Discontinuation of alprazolam treatment in panic patients. American Journal of Psychiatry, 144, 303-308.

Mavissakalian, M. (1982). Pharmacological treatment of anxiety disorders. Journal of Clinical Psychiatry, 43, 487-491.

Nagy, L. M., Krystal, J. H., Woods, S. W., \& Charney, D. S. (1989). Clinical and medication outcome after short-term alprazolam and behavioral group treatment in panic disorder: 2.5-year naturalistic follow-up study. Archives of General Psychiatry, 46, 993-999.

Noyes, R., Garvey, M. J., Cook, B., \& Suelzer, M. (1991). Controlled discontinuation of benzodiazepine treatment for patients with panic disorder. American Journal of Psychiatry, 148, $517-523$.

Noyes, R., Reich, J., Christiansen, J., Suelzer, M., Pfohl, B., \& Coryell, W. A. (1990). Outcome of panic disorder: Relationship to diagnostic subtypes and comorbidity. Archives of General Psychiatry, 47, 809-818.

Pecknold, J. C. (1990). Cross-national collaborative study: Phase I. Journal of Psychiatric Research, 24(Supp. 1), 80-81.

Pecknold, J. C., Swinson, R. P., Kuch, K., \& Lewis, C. P. (1988). Alprazolam in panic disorder and agoraphobia: Results from a multicenter trial. III. Discontinuation effects. Archives of General Psychiatry, 45, 429-436.

Pollack, M. H., Otlo, M. W., Rosenbaum, J. F., Tesar, G. E., Sachs, G. S., \& Cohen, L. S. (1991). Follow-up of clonazepam and alprazolam in panic disorder. Presented at the 144th annual meeting of the American Psychiatric Association, New Orleans, May 14, 1991.

Pollack, M. H., Tesar, G. E., Rosenbaum, J. F., \& Spier, S. A. (1986). Clonazepam in the treatment of panic disorder and agoraphobia: A one-year follow-up. Journal of Clinical Psychopharmacology, 6, 302-304.

Rickels, K. (1990). Discontinuation studies with alprazolam. Journal of Psychiatric Research, 24(Supp. 1), 57-58.

Roy-Byrne, P. P., Uhde, T. W., Post, R. M., Gallucci, W., Chrousos, G. P., \& Gold, P. W. (1986). The corticotropin-releasing hormone stimulation test in patients with panic disorder. American Journal of Psychiatry, 143, 896-899.

Spitzer, R. L., \& Williams, J. B. W. (1986). Structured Clinical Interview for DSM-III-R, Upjohn Version-Revised. Biometrics Research Department, New York State Psychiatric Institute.

Zitrin, C. M., Klein, D. F., \& Woerner, M. G. (1980). Treatment of agoraphobia with group exposure in vivo and imipramine. Archives of General Psychiatry, 37, 63-72. 